\section{Bem-estar psicológico e adolescência: fatores associados}

\author{
Psychological well-being and adolescence: \\ associated factors
}

\footnotetext{
${ }^{1}$ Mestrado em Saúde e Comportamento, Universidade Católica de Pelotas, Pelotas, Brasil. 2 Programa de Pós-graduação em Epidemiologia, Universidade Federal de Pelotas, Pelotas, Brasil.

Correspondência R. A. Silva Mestrado em Saúde e Comportamento, Universidade Católica de Pelotas.

Rua Clóvis Candiota 351, Pelotas, RS 96077-590, Brasil. ricardo.as@uol.com.br
}

\begin{abstract}
This study aimed to evaluate the factors associated with psychological well-being among adolescents in a southern Brazilian city. A crosssectional study was performed with a representative sample $(n=960)$ of adolescents (15-18 years). Eighty-six households were visited in each of the 90 randomized census tracts. Parents signed a written consent form before the adolescent answered a self-reported questionnaire. Psychological well-being was evaluated with a scale containing seven figures representing expressions varying from extreme happiness to extreme sadness. Adolescents were asked to mark the figure that best resembled the way they felt about their lives, and $72.33 \%$ reported a high level of psychological well-being. Prevalence of psychological well-being was higher in families with better economic status and higher maternal schooling. Adolescents who practiced a religion, did not smoke or consume alcohol, and wished to lose weight showed a higher level of psychological well-being, suggesting an interrelationship between health behaviors.
\end{abstract}

Adolescent; Behavior; Cross-Sectional Studies

\author{
Ricardo Azevedo da Silva 1 \\ Bernardo Lessa Horta ${ }^{2}$ \\ Lívia Malta Pontes 1 \\ Augusto Duarte Faria 1 \\ Luciano Dias de Mattos Souza 1 \\ Ana Laura Sica Cruzeiro 1 \\ Ricardo Tavares Pinheiro 1
}

\section{Introdução}

Bem-estar é o grau em que cada pessoa julga a qualidade de sua vida favoravelmente como um todo 1 . A sensação de bem-estar ou de satisfação com a vida é intimamente ligada à forma como o indivíduo é capaz de lidar e absorver a ocorrência de episódios de sua vida, alguns destes inerentes ao próprio curso da mesma. Uma ruptura na sensação de sentir-se psicologicamente bem pode estar associada a um mau manejo de eventos estressantes nas esferas pessoal, social ou cultural afetando comportamentos e influenciando na percepção de saúde do sujeito, trazendo sofrimento pessoal. Entre os eventos estressantes capazes de influenciar a sensação de bem-estar psicológico estão a morte de uma pessoa importante para o indivíduo, desemprego, separação conjugal bem como roubo e doenças na família 2 .

Estudos prévios apontam que o casamento ${ }^{3}$ e a prática religiosa 4,5 estão associados com maior nível de bem-estar psicológico. Essas mesmas variáveis mostram uma tendência para facilitar a recuperação do bem-estar psicológico em indivíduos que passaram por eventos estressantes 6,7. Uma correlação positiva também é encontrada entre bem-estar psicológico e a prática de exercícios físicos 8 .

Não existem dados conclusivos a respeito da relação de bem-estar psicológico e fatores como gênero e idade 2,9 , o que torna relevante a iden- 
tificação de grupos populacionais com índices mais baixos de bem-estar psicológico para que estruture estratégias dirigidas a estas populações, visando melhor sua qualidade de vida.

O objetivo deste estudo foi identificar os fatores associados ao bem-estar psicológico dentro de uma amostra de adolescentes da cidade de Pelotas, Rio Grande do Sul, Brasil.

\section{Metodologia}

Foi realizado um estudo transversal em uma amostra representativa de adolescentes (entre 15 e 18 anos) residentes na zona urbana da cidade de Pelotas no ano de 2002.

A partir dos 448 setores censitários da zona urbana da cidade foram sorteados 90 setores. Em cada um desses setores selecionados, sortearamse um quarteirão e uma esquina como pontos iniciais a partir de onde iniciou-se a visita sistemática de 86 residências. Ao final, 7.740 domicílios haviam sido visitados pelos entrevistadores. O cálculo do tamanho da amostra $(n=800)$ foi feito para um estudo mais amplo sobre comportamentos de saúde em adolescentes do qual o presente trabalho fazia parte.

Após os pais ou responsáveis assinarem um termo de consentimento, o adolescente era convidado a responder um questionário auto-aplicado e sigiloso - com questões sobre nível sócioeconômico, uso de drogas, tabagismo, atividade física, consumo de álcool e uso de métodos anticoncepcionais - que incluía a avaliação de bemestar psicológico, realizada através da escala de faces de Andrews 10. Essa é uma escala visual que contém sete figuras de faces estilizadas representando expressões que variam de extrema felicidade até extrema tristeza. $\mathrm{O}$ adolescente era solicitado a assinalar aquela figura que mais se assemelhava à maneira como ele se sentia a respeito da sua vida. A figura que representava extrema felicidade recebeu valor 1 enquanto a que demonstrava a extrema tristeza o valor 7 . Ou seja: quanto menor o valor declarado maior o grau de bem-estar psicológico.

Na operacionalização do nível sócio-econômico, utilizou-se a Escala de Indicadores de Classe Social da Associação Brasileira de Estudos Populacionais (ABEP) 11 que se baseia na acumulação de bens materiais para classificação em níveis A, B, C, D ou E a partir dos escores alcançados.

Os dados coletados foram processados no programa Epi Info 8.0 (Centers for Disease Control and Prevention, Atlanta, Estados Unidos), com checagem automática da amplitude e consistência. Foram feitas duas digitações que foram posteriormente comparadas para que as incon- sistências fossem resolvidas. A regressão ordinal foi utilizada na análise estatística. Este procedimento não requer a escolha de um ponto de corte arbitrário, como o que acontece na regressão logística. O modelo de odds proporcional foi utilizado. Neste modelo, o odds ratio estima o risco de aumentar em um ponto na escala. Os riscos brutos foram testados para o requisito de proporcionalidade do odds, usando o teste de Brant. Uma vez que apenas quatro adolescentes se consideraram como estando extremamente tristes, houve impossibilidade de atingir o requisito de proporcionalidade do odds. Após esta última categoria ser recodificada para o nível exatamente inferior, obteve-se a proporcionalidade dos odds, permitindo que o modelo de odds proporcional fosse utilizado. Todas as análises controlaram para o efeito do delineamento.

No modelo hierarquizado, cada bloco de variáveis de um determinado nível foi incluído, e as variáveis com um valor $\mathrm{p} \leq 0,20$ no teste de razões de verossimilhança permaneceram no modelo. Nesse tipo de modelo, as variáveis situadas em um nível hierárquico superior ao da variável hierarquicamente inferior são consideradas como potenciais variáveis de confusão da relação entre essa variável e o desfecho em estudo. Já as variáveis situadas em níveis inferiores são consideradas como potenciais mediadores da associação. As variáveis selecionadas em um determinado nível permaneceram nos modelos subseqüentes e foram consideradas como fatores de risco para bem-estar psicológico, mesmo que, com a inclusão de variáveis hierarquicamente inferiores, tivessem perdido sua significância estatística.

O protocolo da presente investigação foi submetido, avaliado e aprovado pelo comitê de ética local, estando de acordo com todos os princípios éticos e legislações vigentes de pesquisas que envolvem seres humanos.

\section{Resultados}

O estudo avaliou uma amostra representativa de 960 adolescentes com idades entre 15 e 18 anos. Dos 1.039 identificados, as perdas ou recusas representaram 7,6\% $(n=79)$ da amostra e ocorreram, em geral, por dois motivos: o adolescente não foi encontrado em casa após, pelo menos, três tentativas ou porque não houve a concordância dos pais ou responsáveis com a realização da entrevista.

Entre os adolescentes entrevistados 89,6\% $(\mathrm{n}=860)$ assinalaram as expressões que representavam os dois maiores níveis de bem-estar psicológico. 
Duas variáveis sócio-econômicas mostraram associação com menores níveis de bem-estar psicológico: baixo nível sócio-econômico e baixa escolaridade dos pais. Na variável baixo nível sócio-econômico aqueles adolescentes cuja família pertencia ao nível sócio-econômico D ou E apresentaram uma chance 1,74 vezes maior (IC95\%: $1,26-2,39)$ de ter um ponto a menos no escore de bem-estar psicológico em relação aos adolescentes de famílias de nível sócio-econômico A ou B. Da mesma forma a variável baixa escolaridade dos pais também esteve associada com uma maior chance de baixa pontuação no escore de bem-estar psicológico (Tabela 1).

Entre as variáveis relacionadas aos comportamentos em saúde dos adolescentes o sedentarismo, tabagismo e a ingestão ou abuso de bebidas alcoólicas no último mês apresentaram associação com pontuações mais baixas de bemestar psicológico. O uso de drogas ilícitas não apresentou associação com bem-estar psicológico, provavelmente por um problema de aferição (a forma como a questão era formulada - "uso de drogas no último mês").

Aqueles adolescentes que relataram pertencer a uma religião apresentaram níveis mais altos de bem-estar psicológico. Ainda dentro das variáveis ligadas ao tema religiosidade, aqueles adolescentes cuja freqüência da prática religiosa era semanal apresentaram níveis maiores de bemestar psicológico do que os que freqüentavam esporadicamente ou nunca. Além disso, adolescentes que acreditavam que Deus costumava ajudá-los apresentaram níveis mais elevados de bem-estar psicológico (Tabela 1).

Na análise multivariada (Tabela 2), nível sócio-econômico e escolaridade materna foram as variáveis localizadas no primeiro nível hierárquico que permaneceram no modelo. Mesmo após controle para escolaridade materna, aqueles adolescentes de famílias de nível sócio-econômico D ou E apresentaram uma chance 1,43 (IC95\%: $1,00-2,06$ ) vezes maior de apresentar um ponto a mais no escore de bem-estar psicológico do que os adolescentes de classe sócio-econômica A ou B. No segundo nível foram incluídas as variáveis ligadas à religiosidade do adolescente, a freqüência com a qual o adolescente reza e a crença de que Deus costuma ajudá-lo, continuaram associados com maior bem-estar psicológico.

Finalmente, no terceiro e último nível incluíram-se as variáveis relacionadas com os comportamentos em saúde do adolescente. Tabagismo, abuso de bebidas alcoólicas no último mês e sedentarismo alcançaram a significância necessária para inclusão no modelo de análise multivariada. A razão de odds para abuso de bebidas alcoólicas aumentou de 1,92 para 2,18 (IC95\%:
1,19-3,98). Enquanto que o efeito do tabagismo e do sedentarismo foi reduzido, após ajuste para fatores de confusão, a associação destas variáveis com o bem-estar psicológico não foi mais estatisticamente significativa.

\section{Discussão}

Este estudo procurou avaliar fatores associados ao bem-estar psicológico em uma amostra populacional de adolescentes. Para isso se utilizou a escala de faces, um instrumento de aplicação simples, apresentado em um formato não verbal que pode ser facilmente assimilável por indivíduos de baixa escolaridade. Uma escala visual como esta registra sentimentos inspirados pela experiência diária e garante que todos os indivíduos sejam estimulados da mesma forma. Isso torna essa categoria de escala um método válido e confiável na aferição de bem-estar psicológico 10.

No presente estudo a grande maioria dos adolescentes entrevistados assinalou as expressões correspondentes aos dois maiores níveis de bem-estar, indicando uma avaliação positiva de sua vida. Embora não tenha sido encontrado estudo sobre este tema com população da mesma faixa etária no Brasil, uma pesquisa de base populacional de maiores de vinte anos utilizandose o mesmo instrumento ${ }^{2}$ encontrou uma prevalência de mal-estar psicológico de $14 \%$. Ainda no mesmo estudo os indivíduos de menor faixa etária demonstraram índices significativamente inferiores de mal-estar psicológico.

Entre a população investigada neste estudo o sentimento de bem-estar psicológico é influenciado pelo nível sócio-econômico. Esse resultado é semelhante ao de um trabalho sobre bem-estar e comportamento saudável conforme status sócio-econômico dos pais 12 que constatou uma maior prevalência de bem-estar psicológico naqueles que se encontram num nível sócio-econômico mais alto.

Os resultados desta investigação mostram que o abuso de álcool diminui o sentimento de bem-estar psicológico entre adolescentes. Aqueles que relataram abuso de álcool no último mês apresentaram menor escore de bem-estar psicológico. Esse dado também foi encontrado em estudo australiano, onde tanto adultos que relataram abuso de álcool como aqueles que se declararam abstêmios apresentaram escores inferiores de bem-estar.

No tocante à religiosidade dos entrevistados, permaneceram as associações entre maior bemestar psicológico e duas variáveis estudadas: freqüência com a qual o adolescente reza e crença 
Razão de odds para escore de bem-estar psicológico (regressão ordinal) de acordo com variáveis sócio-econômicas e demográficas.

\begin{tabular}{|c|c|c|c|}
\hline Variável & $\mathrm{n}$ & OR (IC95\%) & $p$ valor \\
\hline Sexo & & & 0,11 \\
\hline Masculino & 463 & Referência & \\
\hline Feminino & 497 & $1,20(0,96-1,50)$ & \\
\hline Idade (anos) & & & 0,54 * \\
\hline 15 & 227 & Referência & \\
\hline 16 & 271 & $1,34(0,99-1,82)$ & \\
\hline 17 & 221 & $1,40(1,01-1,95)$ & \\
\hline 18 & 241 & $1,11(0,83-1,48)$ & \\
\hline Nível sócio-econômico & & & 0,001 * \\
\hline A ou B & 346 & Referência & \\
\hline C & 366 & $1,32(1,00-1,75)$ & \\
\hline D ou $E$ & 346 & $1,74(1,26-2,39)$ & \\
\hline Escolaridade materna (anos) & & & 0,001 * \\
\hline$\leq 4$ & 198 & $1,61(1,17-2,21)$ & \\
\hline $5-8$ & 416 & $1,56(1,15-2,11)$ & \\
\hline$>8$ & 345 & Referência & \\
\hline Escolaridade paterna (anos) & & & 0,03 \\
\hline$\leq 4$ & 211 & $1,38(1,02-1,85)$ & \\
\hline $5-8$ & 406 & $1,20(0,89-1,61)$ & \\
\hline$>8$ & 343 & Referência & \\
\hline Religião & & & 0,001 \\
\hline Sim & 620 & Referência & \\
\hline Não & 340 & $1,48(1,17-1,86)$ & \\
\hline Freqüência ao culto ou missa & & & 0,008 * \\
\hline Semanalmente & 153 & Referência & \\
\hline Esporadicamente & 295 & $1,19(0,82-1,74)$ & \\
\hline Nunca vai & 512 & $1,57(1,09-2,27)$ & \\
\hline Acreditar que Deus ajuda & & & 0,001 * \\
\hline Bastante & 732 & Referência & \\
\hline Mais ou menos & 155 & $2,20(1,68-2,87)$ & \\
\hline Pouco ou não ajuda & 55 & $1,81(1,01-3,24)$ & \\
\hline Rezar todos os dias & & & 0,001 * \\
\hline Sim & 322 & Referência & \\
\hline Não & 626 & $1,64(1,29-2,07)$ & \\
\hline Sedentarismo & & & 0,007 \\
\hline $\operatorname{Sim}$ & 374 & $1,35(1,09-1,67)$ & \\
\hline Não & 586 & Referência & \\
\hline Consumir bebida alcoólica (último mês) & & & 0,001 \\
\hline $\operatorname{Sim}$ & 410 & $1,49(1,19-1,86)$ & \\
\hline Não & 538 & Referência & \\
\hline Tomar porre (último mês) & & & 0,033 \\
\hline Sim & 61 & $1,92(1,06-3,49)$ & \\
\hline Não & 899 & Referência & \\
\hline Uso de cigarro (cigarros/dia) & & & 0,024 * \\
\hline Não fuma & 769 & Referência & \\
\hline$<5$ & 51 & $1,38(0,86-2,20)$ & \\
\hline $5-15$ & 87 & $1,25(0,80-1,94)$ & \\
\hline$>15$ & 19 & $3,40(1,10-10,49)$ & \\
\hline Uso de drogas ilícitas (último mês) & & & 0,51 \\
\hline $\operatorname{Sim}$ & 83 & $1,17(0,74-1,84)$ & \\
\hline Não & 877 & Referência & \\
\hline
\end{tabular}

* p para tendência. 
Modelo hierárquico final para escore de bem-estar psicológico.

\begin{tabular}{lcc}
\hline Variáveis & OR (IC95\%) & P valor \\
\hline Nível sócio-econômico & & 0,05 * \\
A ou B & Referência & \\
C & $1,15(0,85-1,55)$ & 0,08 * \\
D ou E & $1,43(1,00-2,06)$ & \\
Escolaridade materna (anos) & & 0,001 * \\
$<5$ & $1,34(0,94-1,91)$ & \\
$5-8$ & $1,37(0,98-1,92)$ & \\
$>8$ & Referência & \\
Adolescente acha que Deus ajuda & Referência & \\
Bastante & $2,09(1,60-2,73)$ & \\
Mais ou menos & $1,56(0,86-2,85)$ & \\
Pouco ou nada & & \\
Adolescente reza diariamente & Referência & \\
Sim & $1,40(1,11-1,77)$ & \\
Não & Referência & \\
Tabagismo (cigarros/dia) & $1,07(0,63-1,81)$ & \\
Não fuma & $0,80(0,50-1,26)$ & \\
$<5$ & $2,86(0,93-8,80)$ & \\
$5-15$ & Referência & \\
$>15$ & $2,18(1,19-3,98)$ & \\
Tomou porre no último mês & $1,29,99-1,66)$ & \\
Sim & & \\
Não & & \\
Adolescente sedentário & & \\
Sim & & \\
Não & & \\
\hline
\end{tabular}

de que Deus costuma ajudá-lo. De acordo com estudo anterior 3 os adolescentes que relataram freqüência semanal (ou maior) à igreja apresentaram maiores chances de satisfação com a vida em relação aos que freqüentam a igreja ocasionalmente ou raramente. No mesmo estudo, os jovens que relataram muita religiosidade mostraram maior chance de satisfação com a vida quando comparados com os que declararam religiosidade moderada, discreta ou nenhuma religiosidade. Cabe ressaltar que a associação do bem-estar com a religiosidade pode ainda ter sido influenciada por fatores não avaliados como, por exemplo, dificuldades psicológicas dos entrevistados. Além disso, o delineamento do presente estudo não permite inferências sobre causas e conseqüências do bem-estar psicológico.
Este estudo de base populacional constata uma associação inversamente proporcional entre bem-estar psicológico com nível sócio-econômico e baixa escolaridade materna, indicando que os adolescentes de classes sociais menos favorecidas apresentam menores índices de bemestar psicológico. Uma associação positiva ainda foi encontrada entre o bem-estar psicológico e religiosidade, ou seja, acreditar que Deus ajuda e rezar freqüentemente foram comportamentos que elevaram o bem-estar psicológico do adolescente. Por fim, o abuso de álcool mostrou-se como fator de risco para bem-estar psicológico.

Portanto, programas que visem à redução da pobreza e o controle do uso de álcool entre adolescentes são de extrema importância para a melhoria do bem-estar psicológico. 


\section{Resumo}

Esse trabalho procurou avaliar os fatores associados ao bem-estar psicológico em adolescentes realizando um estudo transversal com amostra representativa $(n=960)$ com idades entre 15 e 18 anos. Foram visitados 86 domicílios em cada um dos 90 setores censitários sorteados. Um consentimento informado era assinado pelos pais ou responsáveis pelo adolescente antes que ele respondesse a um questionário auto-aplicado. O bem-estar psicológico foi avaliado através de uma escala que continha sete figuras que representavam expressões variando de extrema felicidade até extrema tristeza. O adolescente era solicitado a assinalar aquela figura que mais se assemelhava à maneira como se sentia a respeito da sua vida. Entre os entrevistados $72,33 \%$ se consideravam com alto nível de bem-estar psicológico, a prevalência foi maior nas famílias de maior nível sócio-econômico (classe social A ou B) $e$ com maior escolaridade materna. Os resultados também mostraram que pessoas que mantém uma prática religiosa, não fumam, não consomem bebidas alcoólicas e que desejam emagrecer tem maior bem-estar psicológico, sugerindo uma inter-relação entre os comportamentos de saúde.

Adolescente; Comportamento; Estudos Transversais

\section{Referências}

1. Veenhoven R. Questions on happiness; classical topics, modern answers, blidspots. In: Strack F, Argyle M, Schwarz N, editors. Subjective well being: an interdisciplinary perspective. Oxford: Pergamon Press; 1991. p. 7-26.

2. Sparrenberger F, Santos I, Lima RC. Associação de eventos de vida produtores de estresse e mal-estar psicológico: um estudo de base populacional. Cad Saúde Pública 2004; 20:249-58.

3. Hutchinson G, Simeon DT, Bain BC, Wyatt GE, Tucker MB, Lefranc E. Social and health determinants of well being and life satisfaction in Jamaica. Int J Soc Psychiatry 2004; 50:43-53.

4. Koening HG. The Healing power of faith: Science explores medicine's last great frontier. New York: Simon \& Schuster; 1999

5. Matthews DA, Larson DB. Faith and medicine: reconciling the twin traditions of healing. Mind/Body Medicine 1997; 2:3-6.

6. Ellison CG. Religious involvement and subjective well being. J Health Soc Behav 1991; 32:80-99.

7. MacIntosh DN, Silver RC, Wortman CB. Religion's role in adjustment to a negative life event: Coping with the loss of a child. J Pers Soc Psychol 1993; 65:812-21.

\section{Colaboradores}

R. A. Silva concebeu o presente artigo, coordenou a revisão de literatura, realizou, com a colaboração de B. L. Horta e L. D. M. Souza, a análise dos dados e a discussão dos resultados. Os professores B. L. Horta e R. T. Pinheiro foram os autores do projeto e coordenadores gerais do estudo que deu origem ao presente artigo tendo supervisionado o trabalho de campo e a ação dos entrevistadores. A. L. S. Cruzeiro e L. M. Pontes contribuíram em todas as fases do estudo. A. D. Faria e L. D. M. Souza participaram da definição do artigo, revisão de literatura e análise inicial dos dados.
8. Scully D, Kremer J, Meade MM, Graham R, Dudgeon K. Physical exercise and psychological well being: a critical review. Br J Sports Med 1998; 32:111-20.

9. Myers DG. The funds, friends and faith of happy people. Am Psychol 2000; 55:56-67.

10. McDowell I, Newell C. Measuring health: a guide to rating scales and questionnaires. In: McDowell I, Newell C, editors. Psychological well-being. New York: Oxford University Press; 1996. p. 177-236.

11. Associação Brasileira de Estudos Populacionais. Escala de indicadores de classe social. Rio de Janeiro: Associação Brasileira de Estudos Populacionais; 2002.

12. Huurre T, Aro H, Rahkonen O. Well-being and health behaviour by parental socioeconomic status: a follow-up study of adolescents aged 16 until age 32 years. Soc Psychiatry Psychiatr Epidemiol $2003 ; 38: 249-55$

Recebido em 16/Mai/2006

Aprovado em 30/Out/2006 